\title{
ON BOUNDARY ACCUMULATION POINTS OF A CONVEX DOMAIN IN $\mathbb{C}^{n *}$
}

\author{
LINA LEE ${ }^{\dagger}$, BRADLEY THOMAS ${ }^{\ddagger}$, AND BUN WONG§
}

\begin{abstract}
In this paper we show that, for a smoothly bounded convex domain $\Omega \subset \mathbb{C}^{n}$, if there is $\left\{\phi_{j}\right\} \subset$ Aut $(\Omega)$ such that $\phi_{j}(z)$ converges to some boundary point non-tangentially for all $z \in \Omega$, then there does not exist a non-trivial analytic disc on $\partial \Omega$ through any boundary orbit accumulation points.
\end{abstract}

Key words. Automorphism Group, Convex Domains, Invariant Metrics/Measures.

AMS subject classifications. $32 \mathrm{~F} 18,32 \mathrm{~F} 45$.

1. Introduction. The study of biholomorphic automorphism groups, Aut $(\Omega)$, of a domain $\Omega \subset \mathbb{C}^{n}$ is of major interest in various areas of research. The existence of an automorhpism reflects certain symmetry of the domain. It is a deep subject to study the discrete subgroups $\Gamma \subset$ Aut $(\Omega)$ such that $\Omega / \Gamma$ is a compact complex manifold. Although the construction of a cocompact lattice $\Gamma$ in Aut $(\Omega)$ is usually not straightforward, it is comparably easier to find a divergent sequence $\left\{\phi_{j}\right\} \subset \operatorname{Aut}(\Omega)$.

Let $p$ be any point in $\Omega$ such that $\left\{\phi_{j}(p)\right\}$ converge to a boundary point $q \in \partial \Omega$. If we further assume $\partial \Omega$ is smooth, our knowledge of the biholomorphic invariants (i.e., Chern-Moser invariants, invariant Kähler metrics, intrinsic metrics/measures etc.) allows us to draw many interesting conclusions. For instance, if $q \in \partial \Omega$ is strongly pseudoconvex, the method in [9] can be used to show that $\Omega$ must be biholomorphic to the Euclidean ball.

In order to charaterize those smoothly bounded domains with non-compact automorhpism group, it is important to have a better understanding of the orbit accumulation points on the boundary. There has been recently a lot of research in this direction. One of the important conjectures in this regard is due to Greene and Krantz, which can be stated as follows.

Conjecture. Let $\Omega$ be a smoothly bounded domain in $\mathbb{C}^{n}$. Suppose there exists $\left\{\phi_{j}\right\} \subset$ Aut $(\Omega)$ such that $\left\{\phi_{j}(p)\right\}$ accumulates at a boundary point $q \in \partial \Omega$ for some $p \in \Omega$. Then $\partial \Omega$ is of finite type at $q$.

In this paper we will prove the following result in support of the Greene/Krantz conjecture.

ThEOREM. Let $\Omega$ be a smoothly bounded convex domain in $\mathbb{C}^{n}$. Suppose that there is a sequence $\left\{\phi_{j}\right\} \subset$ Aut $(\Omega)$ such that $\left\{\phi_{j}(p)\right\}$ accumulates non-tangentially at some boundary point for all $p \in \Omega$. Then, there does not exist a non-trivial analytic disc on $\partial \Omega$ passing through any orbit accumulation point on the boundary.

In [2] this result was proved in $\mathbb{C}^{2}$ under a more general assumption that $\Omega$ is pseudoconvex. Earlier work in the convex setting in $\mathbb{C}^{2}$ was discussed in $[5,10]$. For

*Received November 9, 2012; accepted for publication September 17, 2013.

${ }^{\dagger}$ Department of Mathematics, University of California, Riverside, CA 92521, USA (linalee@math. ucr.edu).

${ }^{\ddagger}$ Department of Natural and Mathematical Sciences, California Baptist University, Riverside, CA 92504, USA (bthomas@calbaptist.edu).

$\S$ Department of Mathematics, University of California, Riverside, CA 92521, USA (wong@math. ucr.edu). 
the pseudoconvex case, it is a highly non-trivial matter to generalize this result to higher dimensions since the geometry of the boundary of a pseudoconvex domain in $\mathbb{C}^{n}, n>2$, is not as well understood as in $\mathbb{C}^{2}$. To overcome the technical difficulties generalizing the result in $[2,5,9]$, we use the intrinsic measures defined with respect to $U=\mathbb{B}_{n-k} \times \Delta_{k}, 0 \leq k \leq n$, where $\mathbb{B}_{n-k}$ is the unit ball in $\mathbb{C}^{n-k}$ and $\Delta_{k}$ is the unit polydisc in $\mathbb{C}^{k}$. We will prove that the orbit accumulation set on the boundary is actually biholomorphic to a euclidean ball, if it is not a point. This fact allows us to remove the obstacle of finding a higher dimensional analogue of the argument used in [2] for $\mathbb{C}^{2}$, which depends heavily on the classical result that a hyperbolic Riemann surface is covered by the unit disc.

A substantial portion of this paper can be found in [8]; this portion is a joint work of Lina Lee, Bradley Thomas, and Bun Wong.

2. Invariant metrics and invariant measures. Let $H(A, B)$ be the set of holomorphic mappings from $A$ to $B$ and $\Delta$ be the unit disc in $\mathbb{C}$. The Kobayashi and Carathéodory metrics are defined as follows.

Definition 1. The Kobayashi and Carathéodory metrics on $\Omega \subset \mathbb{C}^{n}$ at $p \in \Omega$ in the direction $\xi \in \mathbb{C}^{n}$, denoted as $F_{K}^{\Omega}(p, \xi)$ and $F_{C}^{\Omega}(p, \xi)$, respectively, are defined as follows:

$$
\begin{aligned}
& F_{K}^{\Omega}(p, \xi)=\inf \left\{\frac{1}{\alpha}: \exists \phi \in H(\Delta, \Omega) \text { s.t. } \phi(0)=p, \phi^{\prime}(0)=\alpha \xi\right\} \\
& F_{C}^{\Omega}(p, \xi)=\sup \left\{\left|\sum_{j=1}^{n} \frac{\partial f(p)}{\partial z_{j}} \xi_{j}\right|: \exists f \in H(\Omega, \Delta), \text { s.t. } f(p)=0\right\} .
\end{aligned}
$$

If $z, w \in \Omega$, then the Kobayashi and Carathéodory pseudo-distance on $\Omega$ between $z$ and $w$, denoted as $d_{K}^{\Omega}(z, w)$ and $d_{C}^{\Omega}(z, w)$, respectively, are given by

$$
\begin{aligned}
& d_{K}^{\Omega}(z, w)=\inf _{\gamma} \int_{0}^{1} F_{K}^{\Omega}\left(\gamma(t), \gamma^{\prime}(t)\right) d t, \\
& d_{C}^{\Omega}(z, w)=\sup _{f} \rho(f(z), f(w))
\end{aligned}
$$

where $\gamma:[0,1] \longrightarrow \Omega$ is a piecewise $C^{1}$ curve connecting $z$ and $w$ and $\rho(p, q)$ is the Poincaré distance on $\Delta$ between $p, q \in \Delta$. The supremum in (4) is taken over all holomorphic mappings $f: \Omega \longrightarrow \Delta$.

Kobayashi originally defined the pseudo-distance on $\Omega$ using a chain of analytic discs as follows: for two given points $z, w \in \Omega$, consider a chain of analytic discs $\alpha$ that consists of $z_{1}, z_{2}, \ldots, z_{n} \in \Omega$, analytic discs $f_{i}: \Delta \longrightarrow \Omega$, and $n+1$ pairs of points $a_{0}, b_{0}, a_{1}, b_{1}, \ldots, a_{n}, b_{n} \in \Delta$ such that, for $0 \leq j \leq n$,

$$
f_{j}\left(a_{j}\right)=z_{j}, f_{j}\left(b_{j}\right)=z_{j+1}, \text { and } z_{0}=z, z_{n+1}=w .
$$

We define the length of the chain $\alpha$ as

$$
\ell(\alpha)=\sum_{j=0}^{n} \rho\left(a_{j}, b_{j}\right) .
$$

Then the Kobayashi pseudo-distance between two points $z, w$ is given as

$$
d_{K}^{\Omega}(z, w)=\inf _{\alpha} \ell(\alpha) .
$$


It was Royden [7] who later proved that the definition given by (5) is equivalent to (3).

The metrics and distances given above are invariant under biholomorphic mappings since they satisfy the non-increasing property under holomorphic mappings, i.e., if $\Phi: \Omega_{1} \longrightarrow \Omega_{2}$ is a holomorphic mapping between domains in $\mathbb{C}^{n}$ and $\mathbb{C}^{m}$, respectively, and $p, q \in \Omega_{1}, \xi \in \mathbb{C}^{n}$, then we have

$$
\begin{aligned}
& F^{\Omega_{1}}(p, \xi) \geq F^{\Omega_{2}}\left(\Phi(p), \Phi_{*}(p) \xi\right), \\
& d^{\Omega_{1}}(p, q) \geq d^{\Omega_{2}}(\Phi(p), \Phi(q)),
\end{aligned}
$$

where the metric $F$ in (6) denotes either the Kobayashi or Carathéodory metric and the distance $d$ in (7) is either the Kobayashi or Carathéodory distance.

We extend the definition of the metrics and define the Kobayashi and Carathéodory measures. Let $\mathbb{B}_{k}$ denote the complex $k$-dimensional unit ball and $\Delta_{k}$ the complex $k$-dimensional unit polydisc.

Definition 2. Let $\Omega \subset \mathbb{C}^{n}$ be a domain, $p \in \Omega$, and $\xi_{1}, \ldots, \xi_{m} \in T_{p}^{\mathbb{C}} \Omega, 1 \leq m \leq$ $n$, be linearly independent vectors on the complex tangent space to $\Omega$ at $p$. One can find an $(m, m)$ volume form $M$ on $\Omega$ such that $M\left(\xi_{1}, \ldots, \xi_{m}, \bar{\xi}_{1}, \ldots, \bar{\xi}_{m}\right)=1$. Let $U=\mathbb{B}_{m-j} \times \Delta_{j}, 0 \leq j \leq m$, and $\mu_{m}=\prod_{j=1}^{m}\left(\frac{i}{2} d z_{j} \wedge d \bar{z}_{j}\right)$. We define the Kobayashi and Carathéodory $m$-measures with respect to $U$ as follows:

$$
\begin{gathered}
K_{U}^{\Omega}\left(p ; \xi_{1}, \ldots \xi_{m}\right)=\inf \left\{\frac{1}{\alpha}: \exists \Phi \in H(U, \Omega), \text { s.t. } \Phi(0)=p,\right. \\
\left.\Phi^{*}(0) M=\alpha \mu_{m}, \text { for some } \alpha>0\right\}, \\
C_{U}^{\Omega}\left(p ; \xi_{1}, \ldots, \xi_{m}\right)= \\
\sup \{\beta: \exists \Phi \in H(\Omega, U), \text { s.t. } \Phi(p)=0, \\
\left.\Phi^{*}(p) \mu_{m}=\beta M, \beta>0\right\} .
\end{gathered}
$$

The Kobayashi and Carathéodory measures satisfiy the non-increasing property under holomorphic mappings.

Proposition 1. Let $\Omega_{1} \subset \mathbb{C}^{n}, \Omega_{2} \subset \mathbb{C}^{n^{\prime}}$ be domains and $U=\mathbb{B}_{m-j} \times \Delta_{j}$, $0 \leq j \leq m, m \leq \min \left\{n, n^{\prime}\right\}$. Let $p \in \Omega_{1}, \xi_{j} \in T_{p}^{\mathbb{C}} \Omega_{1}, j=1, \ldots, m$, and $\xi_{j}$ 's be linearly independent. If $\phi \in H\left(\Omega_{1}, \Omega_{2}\right)$ is such that $\phi_{*}(p) \xi_{j}$ 's are linearly independent, then

$$
\begin{aligned}
K_{U}^{\Omega_{1}}\left(p ; \xi_{1}, \ldots \xi_{m}\right) & \geq K_{U}^{\Omega_{2}}\left(\phi(p) ; \phi_{*}(p) \xi_{1}, \ldots \phi_{*}(p) \xi_{m}\right), \quad \text { and } \\
C_{U}^{\Omega_{1}}\left(p ; \xi_{1}, \ldots, \xi_{m}\right) & \geq C_{U}^{\Omega_{2}}\left(\phi(p) ; \phi_{*}(p) \xi_{1}, \ldots \phi_{*}(p) \xi_{m}\right) .
\end{aligned}
$$

Proof. Let $M$ be an $(m, m)$ volume form on $\Omega_{1}$ such that $M\left(\xi_{1}, \ldots, \xi_{m}, \bar{\xi}_{1}, \ldots, \bar{\xi}_{m}\right)=1$. Let $\Phi: U \longrightarrow \Omega_{1}$ be a holomorphic mapping such that $\Phi(0)=p, \Phi^{*}(0) M=\alpha \mu_{m}$. Consider $h=\phi \circ \Phi: U \longrightarrow \Omega_{2}$. Let $M^{\prime}$ be an $(m, m)$ volume form on $\Omega_{2}$ such that $\phi^{*}(p) M^{\prime}=M$. Then $h(0)=\phi(p)$ and

$$
h^{*}(0) M^{\prime}=\Phi^{*}(0)\left(\phi^{*}(p) M^{\prime}\right)=\Phi^{*}(0)(M)=\alpha \mu_{m} .
$$

Hence $1 / \alpha \geq K_{U}^{\Omega_{2}}(\phi(p), M)$ and $\inf 1 / \alpha \geq K_{U}^{\Omega_{2}}(\phi(p), M)$. One can show the second inequality in a similar way. 
Corollary 1. Let $\Omega_{1}, \Omega_{2} \subset \mathbb{C}^{n}$ be domains and $U=\mathbb{B}_{m-j} \times \Delta_{j}, 0 \leq j \leq m$, $m \leq n$. Let $p \in \Omega_{1}, \xi_{j} \in T_{p}^{\mathbb{C}} \Omega_{1}, j=1, \ldots, m$, and $\xi_{j}$ 's be linearly independent. If $\phi: \Omega_{1} \longrightarrow \Omega_{2}$ is a biholomorphism, we have

$$
\begin{aligned}
K_{U}^{\Omega_{1}}\left(p ; \xi_{1}, \ldots \xi_{m}\right) & =K_{U}^{\Omega_{2}}\left(\phi(p) ; \phi_{*}(p) \xi_{1}, \ldots \phi_{*}(p) \xi_{m}\right), \quad \text { and } \\
C_{U}^{\Omega_{1}}\left(p ; \xi_{1}, \ldots, \xi_{m}\right) & =C_{U}^{\Omega_{2}}\left(\phi(p) ; \phi_{*}(p) \xi_{1}, \ldots \phi_{*}(p) \xi_{m}\right) .
\end{aligned}
$$

Proof. Proposition 1 holds for $\phi$ and $\phi^{-1}$. Therefore we have inequalities in both directions.

Corollary 2. Let $U=\mathbb{B}_{m-j} \times \Delta_{j}, p \in U, \xi_{j} \in T_{p}^{\mathbb{C}} U, 1 \leq j \leq m$, and $\xi_{j}$ 's be linearly independent vectors. We have $K_{U}^{U}\left(p ; \xi_{1}, \ldots \xi_{m}\right)=C_{U}^{U}\left(p ; \xi_{1}, \ldots, \xi_{m}\right)$ for all $p \in U$.

Proof. Since the automorphism group on $U$ is transitive, we may assume $p=0$. Also we may assume $\mu_{m}\left(\xi_{1}, \ldots, \xi_{m}, \bar{\xi}_{1}, \ldots, \bar{\xi}_{m}\right)=1$. Let $f \in H(U, U)$ be such that $f(0)=0$ and that $f^{*}(0) \mu_{m}=\alpha \mu_{m}, \alpha>0$. By Carathéodory-Cartan-Kaup-Wu theorem, we have $\alpha \leq 1$. Since one can choose $f$ as the identity mapping, we have $\inf 1 / \alpha=1=\sup \alpha$. Therefore $K_{U}^{U}\left(0, \mu_{m}\right)=C_{U}^{U}\left(0, \mu_{m}\right)=1$. The automorphism group on $U$ is transitive. Hence by Corollary 1 we have $K_{U}^{U}\left(p, \mu_{m}\right)=C_{U}^{U}\left(p, \mu_{m}\right)$ for any $p \in U$.

Proposition 2. Let $\Omega \subset \mathbb{C}^{n}, p \in \Omega$ and $\xi_{1}, \ldots, \xi_{m} \in T_{p}^{\mathbb{C}} \Omega, 1 \leq m \leq n$ be linearly independent vectors. If $U=\mathbb{B}_{m-j} \times \Delta_{j}, 0 \leq j \leq m$, then

$$
\frac{C_{U}^{\Omega}\left(p, \xi_{1}, \ldots, \xi_{m}\right)}{K_{U}^{\Omega}\left(p, \xi_{1}, \ldots, \xi_{m}\right)} \leq 1 .
$$

Proof. Let $M$ be an $(m, m)$ volume form on $\Omega$ such that $M\left(\xi_{1}, \ldots, \xi_{m}, \bar{\xi}_{1}, \ldots, \bar{\xi}_{m}\right)=1$. Let $\Phi: U \longrightarrow \Omega$ be a holomorphic mapping such that $\Phi(0)=p, \Phi^{*}(0) M=\alpha \mu_{m}, \alpha>0$ and $\Psi: \Omega \longrightarrow U$ be a holomorphic mapping such that $\Psi(p)=0, \Psi^{*}(p) \mu_{m}=\beta M$. Consider $h=\Psi \circ \Phi: U \longrightarrow U$. Then $h(0)=0$ and $h^{*}(0) \mu_{m}=\alpha \cdot \beta \cdot \mu_{m}$. By Carathéodory-Cartan-Kaup-Wu theorem we have $\alpha \cdot \beta \leq 1$. Hence $\beta \leq 1 / \alpha$. The inequality (8) follows after taking the infimum over $\alpha$ 's and the supremum over $\beta$ 's. [

Lemma 1. Let $\Omega \subset \mathbb{C}^{n}, p \in \Omega$ and $\xi_{1}, \ldots, \xi_{m} \in T_{p}^{\mathbb{C}} \Omega, 1 \leq m \leq n$, be linearly independent vectors. Let $U=\mathbb{B}_{m-j} \times \Delta_{j}$. We have $\frac{C_{U}^{\Omega}\left(p ; \xi_{1}, \ldots, \xi_{m}\right)}{K_{U}^{\Omega}\left(p ; \xi_{1}, \ldots, \xi_{m}\right)}=1$ if and only if $\Omega$ is biholomorphic to $U$.

Proof. One can use a similar argument as in [9] (Theorem E).

The Kobayashi $m$-measure is localizable near a strongly pseudocovnex boundary point. Refer to [6] for a detailed explanation. The Carathéodory $m$-measure is localizable near a boundary point $p$ if one can find a global peak function that peaks at $p$. Hence we have the following Lemma.

Lemma 2. Let $\Omega \subset \mathbb{C}^{n}$ be a smoothly bounded convex domain and $p \in \partial \Omega$ be a strongly covnex boundary point. Let $V$ be a neighborhood of $p$. Then we have

$$
\frac{K_{U}^{\Omega}\left(z ; \xi_{1}, \ldots, \xi_{m}\right)}{K_{U}^{\Omega \cap V}\left(z ; \xi_{1}, \ldots, \xi_{m}\right)} \rightarrow 1, \quad \frac{C_{U}^{\Omega}\left(z ; \xi_{1}, \ldots, \xi_{m}\right)}{C_{U}^{\Omega \cap V}\left(z ; \xi_{1}, \ldots, \xi_{m}\right)} \rightarrow 1, \quad \text { as } z \rightarrow p .
$$


REMARK 1. Let $\Omega$ be a smoothly bounded convex domain. The domain $\Omega$ near a strongly convex boundary point can be approximated by ellipsoids which are biholomorphic to balls. Since $\mathbb{B}_{m}$ and $\mathbb{B}_{m-j} \times \Delta_{j}, j \geq 1$, are not biholomorphic and the Kobayashi and Carathéodory measures are localizable near a strongly convex boundary point by Lemma 2, we have

$$
\begin{aligned}
& \frac{C_{U}^{\Omega}\left(z ; \xi_{1}, \ldots, \xi_{m}\right)}{K_{U}^{\Omega}\left(z ; \xi_{1}, \ldots, \xi_{m}\right)}<c<1, U=\mathbb{B}_{m-j} \times \Delta_{j}, j \geq 1 \\
& \frac{C_{U}^{\Omega}\left(z ; \xi_{1}, \ldots, \xi_{m}\right)}{K_{U}^{\Omega}\left(z ; \xi_{1}, \ldots, \xi_{m}\right)} \rightarrow 1, \quad U=\mathbb{B}_{m}
\end{aligned}
$$

as $z$ approaches a strongly convex boundary point.

\section{Geometry of a convex domain.}

3.1. Non-tangential convergence. Let $\Omega \subset \mathbb{C}^{n}$ be a domain with a $C^{1}$ boundary. Let $\left\{q_{j}\right\} \subset \Omega$ be a sequence of points. We say $q_{j} \rightarrow q \in \partial \Omega$ non-tangentially for some boundary point $q$ if

$$
q_{j} \in \Gamma_{\alpha}(q)=\{z \in \Omega:|z-q|<\alpha \operatorname{dist}(z, \partial \Omega)\}
$$

for all $j$ large enough for some $\alpha>1$ and we say $q_{j} \rightarrow q \in \partial \Omega$ normally if $q_{j}$ 's approach $q$ along the real normal line to the boundary through $q$ for all $j$ large enough.

Lemma 3. Let $\Omega \subset \mathbb{C}^{n}$ be a convex domain with $C^{1}$ boundary and $q \in \partial \Omega$. Let $\nu$ be the outward unit normal vector to $\partial \Omega$ at $q$ and $q^{\prime}=q-t \nu \in \Omega$ for some small $t>0$. Then we have

$$
\Gamma_{\alpha}(q) \subset\left\{z \in \Omega: 0 \leq \angle z q q^{\prime}<\arccos (1 / \alpha)\right\}
$$

Proof. Let $q=0$ and $\nu=(0, \ldots, 0,1)$. Then $\Omega \subset H=\left\{\operatorname{Re} z_{n}<0\right\}$. Therefore $\operatorname{dist}(z, \partial \Omega) \leq \operatorname{dist}(z, \partial H)=\left|\operatorname{Re} z_{n}\right|$. Hence $|z-q|<\alpha\left|\operatorname{Re} z_{n}\right|=\alpha\left|\left(0, \ldots, 0, \operatorname{Re} z_{n}\right)\right|$. Therefore $\angle z q q^{\prime}<\arccos (1 / \alpha)$.

Lemma 4. Let $\Omega \subset \mathbb{C}^{n}$ be a convex domain with $C^{1}$ boundary. Suppose $\left\{\phi_{j}\right\} \subset$ Aut $(\Omega)$ and $\phi_{j}(p) \rightarrow q \in \partial \Omega$ non-tangentially for some $p \in \Omega$. Then there exists $\left\{p_{j}\right\} \subset \Omega$ such that $\phi_{j}\left(p_{j}\right) \rightarrow q$ normally and that $d_{K}^{\Omega}\left(p, p_{j}\right) \leq r$ for some $r>0$.

Proof. Let $\phi_{j}(p)=q_{j}$. Since $q_{j} \rightarrow q$ non-tangentially, one can find $\alpha>1$ such that $q_{j} \in \Gamma_{\alpha}(q)$ for all $j$ large enough.

Let $\nu$ be the outward unit normal vector to $\partial \Omega$ at $q$ and $\ell_{q}$ be the real normal line to $\partial \Omega$ through $q$, i.e., $\ell_{q}=\{q+t \nu: t \in \mathbb{R}\}$. Define the mapping $\pi: \Omega \longrightarrow \ell_{q}$ as the projection of $\Omega$ onto $\ell_{q}$. Let $\tilde{q_{j}}=\pi\left(q_{j}\right)$. Then we have $\left|q_{j}-\tilde{q}_{j}\right| \leq\left|q-q_{j}\right|<$ $\alpha \operatorname{dist}\left(q_{j}, \partial \Omega\right)$. Let $p_{j}=\phi_{j}^{-1}\left(\tilde{q}_{j}\right)$. Then we have $\phi_{j}\left(p_{j}\right)=\tilde{q}_{j} \rightarrow q$ normally after taking a subsequence if necessary.

Since $\Omega$ is convex, by Lemma 3 , we have $0 \leq \angle q_{j} q \tilde{q_{j}} \leq \arccos (1 / \alpha)$. Therefore

$$
\cos \left(\angle q_{j} q \tilde{q}_{j}\right)=\frac{\left|\tilde{q_{j}}-q\right|}{\left|q_{j}-q\right|} \geq \frac{1}{\alpha}
$$


Let $\gamma(t)=(1-t) q_{j}+t \tilde{q_{j}}$. Then we have

$$
\begin{aligned}
d_{K}^{\Omega}\left(p, p_{j}\right) & =d_{K}^{\Omega}\left(q_{j}, \tilde{q}_{j}\right) \leq \int_{0}^{1} F_{K}\left(\gamma(t), \gamma^{\prime}(t)\right) d t \\
& \leq \int_{0}^{1}\left|\gamma^{\prime}(t)\right| \frac{1}{\operatorname{dist}(\gamma(t), \partial \Omega)} d t \leq \int_{0}^{1}\left|\gamma^{\prime}(t)\right| \frac{\alpha}{|\gamma(t)-q|} d t \\
& \leq \frac{\alpha\left|q_{j}-\tilde{q}_{j}\right|}{\left|\tilde{q}_{j}-q\right|} \leq \frac{\alpha\left|q_{j}-q\right|}{\left|\tilde{q}_{j}-q\right|} \leq \alpha^{2}
\end{aligned}
$$

We let $r=\alpha^{2}$.

Lemma 5. Let $\Omega \subset \subset \mathbb{C}^{n}$ be a bounded complete hyperbolic domain with a $C^{2}$ boundary and $p \in \partial \Omega$ be a strongly convex boundary point. Then for any fixed $r>0$, the Euclidean diam $\beta_{K}^{\Omega}(z, r) \longrightarrow 0$ as $z \rightarrow p$, where

$$
\beta_{K}^{\Omega}(z, r)=\left\{w \in \Omega: d_{K}^{\Omega}(z, w)<r\right\} \subset \Omega .
$$

Proof. Let $\delta(z)=\operatorname{dist}(z, \partial \Omega)$ and $z^{\prime} \in \Omega$ be the boundary point that satisfies $\left|z-z^{\prime}\right|=\delta(z)$. It is a well-known fact that for $z \in \Omega$ close to a strongly pseudoconvex boundary point the Kobayashi metric estimate is given as follows (refer to $[1,3]$ ):

$$
F_{K}^{\Omega}(z, \xi) \approx \frac{1}{\delta(z)} \xi_{N}+\frac{1}{\sqrt{\delta(z)}} \xi_{T},
$$

where $\xi_{T}$ and $\xi_{N}$ are the tangential and normal components of $\xi$ at $z^{\prime}$, respectively. The assertion can be derived from the above fact and the complete hyperbolicity.

3.2. Maximal chain of analytic discs. Let $\Omega \subset \mathbb{C}^{n}$ be a smoothly bounded domain and $V$ be a connected subset of $\partial \Omega$. We say $\partial \Omega$ is geometrically flat along $V$ if the direction of the gradient vector of $\partial \Omega$ does not change along $V$.

The following proposition is the generalization of Lemma 3.2 in [10]. The proof is basically the same.

Proposition 3. Let $\Omega \subset \subset \mathbb{C}^{n}$ be a bounded convex domain. If $\phi: \Delta \longrightarrow \partial \Omega$ is a holomorphic mapping, then $\partial \Omega$ is geometrically flat along $\phi(\Delta)$.

Proof. Let $\Omega=\{\rho<0\}$ and $p=\phi(0) \in \partial \Omega$. Let $H=\{\operatorname{Re} h=0\}$ be the real tangent plane to $\partial \Omega$ at $p$, where $h$ is a linear holomorpic function. Since $\Omega$ is convex, we have $\bar{\Omega} \subset\{\operatorname{Re} h \leq 0\}$. Consider $f(\zeta)=h \circ \phi(\zeta)$. Then $f$ is a holomorphic function on $\Delta$ and satisfies $\operatorname{Re} f(\zeta) \leq 0$ for all $\zeta \in \Delta$ and that $\operatorname{Re} f(0)=0$. By the maximum principle for harmonic functions, we have $\operatorname{Re} f(\zeta)=0$ for all $\zeta \in \Delta$. Therefore $f \equiv 0$ on $\Delta$ and hence $h \equiv 0$ on $\phi(\Delta)$.

Definition 3. Let $H \subset \mathbb{C}^{n}$ be a subset of $\mathbb{C}^{n}$ and $q \in H$. We define the maximal chain of analytic discs on $H$ through $q$, denoted as $\Delta_{q}^{H}$, as follows:

$$
\Delta_{q}^{H}=\{z \in H \text { : there exists a finite chain of analytic discs joining } z \text { and } q\}
$$

i.e., there exists holomorphic maps $\phi_{1}, \phi_{2}, \ldots, \phi_{k}: \Delta \longrightarrow \mathbb{C}^{n}$ such that $\phi_{j}(\Delta) \subset H$, $1 \leq j \leq k$, and $z_{i} \in H, a_{i}, b_{i} \in \Delta, 1 \leq i \leq k$, such that $\phi_{j}\left(a_{j}\right)=z_{j-1}, \phi_{j}\left(b_{j}\right)=z_{j}$, where $z_{0}=q$ and $z_{k}=z$. Note that $\Delta_{q}^{H}=\Delta_{z}^{H}$, if $z \in \Delta_{q}^{H}$. We say $\Delta_{q}^{H}$ is trivial if $\Delta_{q}^{H}=\{q\}$. 
REMARK 2. If $V \subset H$ is a complex variety through $q$, then $V \subset \Delta_{q}^{H}$.

The following Corollary follows immediately from Proposition 3.

Corollary 3. If $\Omega \subset \subset \mathbb{C}^{n}$ is a smoothly bounded convex domain, then $\partial \Omega$ is geometrically flat along $\Delta_{q}^{\partial \Omega}$ for all $q \in \partial \Omega$.

In the following theorem we show that a maximal chain of analytic discs on the boundary of a smoothly bounded convex domain is linearly convex.

TheOREM 1. Let $\Omega \subset \subset \mathbb{C}^{n}$ be a smoothly bounded convex domain. Then $\Delta_{q}^{\partial \Omega}$ is linearly convex for all $q \in \partial \Omega$, i.e., if $z, w \in \Delta_{q}^{\partial \Omega}$, then $t \cdot z+(1-t) w \in \Delta_{q}^{\partial \Omega}$ for all $t \in[0,1]$.

Proof. We first show that if $z, w \in \Delta_{q}^{\partial \Omega}$, then $t \cdot z+(1-t) w \in \partial \Omega$ for all $t \in$ $[0,1]$. Since $\partial \Omega$ is geometrically flat along $\Delta_{q}^{\partial \Omega}$, we may assume $\Delta_{q}^{\partial \Omega} \subset\left\{\operatorname{Re} z_{n}=0\right\}$. We have $t \cdot z+(1-t) w \in \bar{\Omega}$ since $\Omega$ is convex. Also $\operatorname{Re}(t \cdot z+(1-t) w)_{n}=t$. $\operatorname{Re} z_{n}+(1-t) \operatorname{Re} w_{n}=0$ for all $t \in[0,1]$. Since $\operatorname{Re} z_{n}<0$ for all $z \in \Omega$, we have $t \cdot z+(1-t) w \in \partial \Omega$.

We use induction on the length of the chain (i.e. number of analytic discs) joining two points $z, w \in \Delta_{q}^{\partial \Omega}$.

Suppose $z, w \in \Delta_{q}^{\partial \Omega}$ and $z, w$ both lie on the same analytic disc, then $t \cdot z+$ $(1-t) w \in \Delta_{q}^{\partial \Omega}$. Let $z=\phi(a)$ and $w=\phi(b)$ for some analytic disc $\phi: \Delta \longrightarrow \partial \Omega$ and $a, b \in \Delta$ and define an analytic disc $\tilde{\phi}_{t}$ as follows:

$$
\tilde{\phi}_{t}(\zeta)=t \cdot \phi(\zeta)+(1-t) \phi(b) .
$$

Then $\tilde{\phi}_{t}(\zeta) \in \partial \Omega$ for all $\zeta \in \Delta$ and for any fixed $t \in[0,1]$, and $\tilde{\phi}_{t}(b)=\phi(b) \in \Delta_{q}^{\partial \Omega}$. Hence $\tilde{\phi}_{t}(\zeta) \in \Delta_{q}^{\partial \Omega}$ for all $\zeta \in \Delta$. Therefore $\tilde{\phi}_{t}(a)=t \cdot \phi(a)+(1-t) \phi(b) \in \Delta_{q}^{\partial \Omega}$ for all $t \in[0,1]$.

Assume $t \cdot z+(1-t) w \in \Delta_{q}^{\partial \Omega}$ for all $t \in[0,1]$ if $z, w$ can be joined by a chain of length less than or equal to $n$. Suppose $z, w \in \Delta_{q}^{\partial \Omega}$ can be joined by $n+1$ number of analytic discs, i.e., there exists analytic discs $\phi_{j}: \Delta \longrightarrow \partial \Omega, a_{j}, b_{j} \in \Delta$ and $z_{j} \in \partial \Omega$, $1 \leq j \leq n+1$, such that $\phi_{j}\left(a_{j}\right)=z_{j-1}, \phi_{j}\left(b_{j}\right)=z_{j}$ and $z=z_{0}, w=z_{n+1}$. Define an analytic $\operatorname{disc} \tilde{\phi}_{t}$ as follows:

$$
\tilde{\phi}_{t}(\zeta)=t \cdot \phi_{1}(\zeta)+(1-t) \phi_{n+1}\left(b_{n+1}\right), \quad t \in[0,1] .
$$

Then $\tilde{\phi}_{t}(\zeta) \in \partial \Omega$ for all $\zeta \in \Delta$ and for all $t \in[0,1]$. We have

$$
\begin{aligned}
\tilde{\phi}_{t}\left(b_{1}\right) & =t \cdot \phi_{1}\left(b_{1}\right)+(1-t) \phi_{n+1}\left(b_{n+1}\right) \\
& =t \cdot \phi_{2}\left(a_{2}\right)+(1-t) \phi_{n+1}\left(b_{n+1}\right)
\end{aligned}
$$

and hence $\tilde{\phi}_{t}\left(b_{1}\right) \in \Delta_{q}^{\partial \Omega}$ for all $t \in[0,1]$ since $\phi_{2}\left(a_{2}\right)$ and $\phi_{n+1}\left(b_{n+1}\right)$ are joined by $n$ analytic discs. Therefore $\tilde{\phi}_{t}(\zeta) \in \Delta_{q}^{\partial \Omega}$ for all $\zeta \in \Delta$ and hence $\tilde{\phi}_{t}\left(a_{1}\right)=$ $t \cdot z+(1-t) w \in \Delta_{q}^{\partial \Omega}$ for all $t \in[0,1]$

\section{Normal convergence.}

Proposition 4. Let $\Omega$ be a smoothly bounded convex domain in $\mathbb{C}^{n}$. Suppose $\left\{\phi_{j}\right\} \subset \operatorname{Aut}(\Omega)$ and $\phi_{j}(p) \rightarrow q \in \partial \Omega$ non-tangentially for some $p \in \Omega$ and that $\Delta_{q}^{\partial \Omega}$ is not trivial. Then there exists a non-constant holomorphic onto mapping $\phi: \Omega \longrightarrow$ $\Delta_{q}^{\partial \Omega}$ such that $\phi_{j} \rightarrow \phi$ after taking a subsequence if necessary. 
Proof. Since $\phi_{j}(p) \rightarrow q \in \partial \Omega$, we konw that $\phi_{j} \rightarrow \phi$ locally uniformly (after taking a subsequence if necessary) where $\phi: \Omega \longrightarrow \partial \Omega$ is a holomorphic mapping by a normal family argument.

We shall show that $\phi(\Omega)=\Delta_{q}^{\partial \Omega}$. Since $\phi(\Omega) \subset \Delta_{q}^{\partial \Omega}$ is clear, we need only to show that $\Delta_{q}^{\partial \Omega} \subset \phi(\Omega)$.

Let $q^{\prime} \in \Delta_{q}^{\partial \Omega}$ and $q^{\prime} \neq q$. By Corollary $3, \partial \Omega$ is geometrically flat along $\Delta_{q}^{\partial \Omega}$. Let $\nu$ be the constant outward unit normal vector to $\partial \Omega$ along $\Delta_{q}^{\partial \Omega}$. By Lemma 4, there exists $\left\{p_{j}\right\} \subset \beta_{K}^{\Omega}(p, r)$ for some $r>0$ such that $\phi_{j}\left(p_{j}\right) \rightarrow q$ normally. Let $\delta_{j}$ 's be such that

$$
\phi_{j}\left(p_{j}\right)=q-\delta_{j} \nu
$$

Then we have

$$
d_{K}^{\Omega}\left(q-\delta_{j} \nu, q^{\prime}-\delta_{j} \nu\right)<r^{\prime}<\infty,
$$

for all $j$ for some $r^{\prime}>0$. Hence if we let $p_{j}^{\prime}=\phi_{j}^{-1}\left(q^{\prime}-\delta_{j} \nu\right)$, then

$$
\begin{aligned}
d_{K}^{\Omega}\left(p_{j}^{\prime}, p\right) & \leq d_{K}^{\Omega}\left(p_{j}, p_{j}^{\prime}\right)+d_{K}^{\Omega}\left(p_{j}, p\right) \\
& =d_{K}^{\Omega}\left(q-\delta_{j} \nu, q^{\prime}-\delta_{j} \nu\right)+r<r+r^{\prime}<\infty, \quad \forall j .
\end{aligned}
$$

Since $\overline{\beta_{K}^{\Omega}\left(p, r+r^{\prime}\right)}$ is compact in $\Omega$, one can find $p^{\prime} \in \Omega$ such that $p_{j}^{\prime} \rightarrow p^{\prime}$ and that $\phi\left(p^{\prime}\right)=q^{\prime}$. Therefore $\Delta_{q}^{\partial \Omega} \subset \phi(\Omega)$.

Corollary 4. Let $\Omega \subset \subset \mathbb{C}^{n}$ be a smoothly bounded convex domain and $\left\{\phi_{j}\right\} \subset$ Aut $(\Omega)$. If $\phi_{j}(p) \rightarrow q \in \partial \Omega$ non-tangentially and $\Delta_{q}^{\partial \Omega}$ is not trivial, then $\Delta_{q}^{\partial \Omega}$ is an open convex set contained in a complex $m$-dimensional plane, where $m=\operatorname{dim}_{\mathbb{C}} \Delta_{q}^{\partial \Omega}$.

Proof. By Theorem 1, $\Delta_{q}^{\partial \Omega}$ is convex. Hence it is contained in a complex $m$ dimensional plane, where $m=\operatorname{dim}_{\mathbb{C}} \Delta_{q}^{\partial \Omega}$. Suppose $\Delta_{q}^{\partial \Omega}$ is not open and $w \in \partial \Delta_{q}^{\partial \Omega}$ is a boundary point. By Proposition 4, one can find $z \in \Omega$ such that $\phi(z)=w$, where $\phi$ is the limit of $\left\{\phi_{j}\right\}$. One can find a germ of complex $m$-dimensional manifold, say $M$, near $z$ such that $\operatorname{dim}_{\mathbb{C}} \phi(M)=m$. Let $H$ be the complex $m-1$ dimensional subspace of the real supporting plane to $\Delta_{q}^{\partial \Omega}$ at $w=\phi(z)$. By the maximum principle argument used in Proposition 3, we have that $\phi(M) \subset H$. But $\operatorname{dim} H<m$. Hence a contradiction.

TheOREM 2. Let $\Omega \subset \subset \mathbb{C}^{n}$ be a smoothly bounded domain. Suppose $\Delta_{q}^{\partial \Omega}$ is not trivial for some $q \in \partial \Omega$ and that $\phi: \Omega \longrightarrow \Delta_{q}^{\partial \Omega}$ is a surjective holomorphic mapping. Then there exists a sequence of points $\left\{p_{j}\right\} \subset \Omega$ such that $p_{j} \rightarrow p \in \partial \Omega$ and that $\left\{\phi\left(p_{j}\right)\right\} \subset \Delta_{q}^{\partial \Omega}$ converge to a point in $\Delta_{q}^{\partial \Omega}$ for some strongly pseudoconvex boundary point $p \in \partial \Omega$.

Proof. Since $\Omega$ is smoothly bounded, there exists a strongly pseudoconvex boundary point $p \in \partial \Omega$. Let $\nu$ be the outward unit normal vector to $\partial \Omega$ at $p$. One can find a holomorphic support function $h$ of $\partial \Omega$ at $p$ such that, for a small neighborhood $U$ of $p$, we have $\{h=0\} \cap \bar{\Omega} \cap U=\{p\}$. Let $H=\{h=0\}$ and let $H_{n}$ be the translation of $H$ in the direction of $-\nu$ by the length of $1 / n$, i.e.,

$$
H_{n}=\left\{z-\nu \frac{1}{n}: z \in H\right\}, \quad n \in \mathbb{N} .
$$


One can find a small neighborhood $U$ of $p$ and $N>0$ large enough such that $\partial \Omega \cap U$ is strongly pseudoconvex and that $H_{n} \cap \Omega \subset U \cap \Omega$ for all $n>N$.

Let $\operatorname{dim}_{\mathbb{C}} \Delta_{q}^{\partial \Omega}=m$. Choose a complex $m$-dimensional closed analytic subset of $H_{n}$ through $p-\nu \cdot \frac{1}{n}$ and perturb it at $p-\nu \cdot \frac{1}{n}$, call it $H_{n}^{\prime}$, so that the rank of the restriction mapping of $\phi$ on $H_{n}^{\prime}$, say $\phi_{n}: H_{n}^{\prime} \stackrel{{ }_{n}}{\longrightarrow} \Delta_{q}^{\partial \Omega}$, has rank $m$ generically and that $\partial H_{n}^{\prime} \subset \partial \Omega$. One can make the perturbation small enough that $H_{n}^{\prime} \subset U \cap \Omega$ for all $n$. Suppose $\phi_{n}$ is not proper for some $n>N$. Then one can find a compact set $K \subset \subset \Delta_{q}^{\partial \Omega}$ such that the preimage of $K$ is not compact in $H_{n}^{\prime}$. Hence one can find $\left\{p_{j}\right\} \subset H_{n}^{\prime}$ such that $\phi\left(p_{j}\right)$ 's lie in $K$ for all $j$ and $p_{j}$ 's approach a boundary point of $H_{n}^{\prime}$, which is strongly pseudoconvex.

If $\phi_{n}$ is proper for all $n>N$, then they are surjective because the rank of $\phi_{n}$ is equal to $m$. One can find $p_{n} \in H_{n}^{\prime}$ for $n>N$, arbitrarily close to $p$, which is a strongly pseudoconvex point. Moreover $\left\{\phi\left(p_{n}\right)\right\}$ converge to a point in $\Delta_{q}^{\partial \Omega}$.

Lemma 6. Let $\Omega \subset \subset \mathbb{C}^{n}$ be a smoothly bounded convex domain. Suppose there exists $\left\{\phi_{j}\right\} \subset$ Aut $(\Omega)$ such that $\phi_{j}(z)$ converges to some boundary point non-tangentially for all $z \in \Omega$ for some fixed $\alpha$ in (9) and that $\Delta_{q}^{\partial \Omega}$ is not trivial for some orbit accumulation point $q \in \partial \Omega$. Then for any $\epsilon>0$ one can find $\left\{p_{j}\right\} \subset \Omega$ such that $\phi_{j}\left(p_{j}\right) \rightarrow q^{\prime} \in \Delta_{q}^{\partial \Omega}$ normally for some point $q^{\prime}$ and that $p_{j} \in B\left(p^{\prime}, \epsilon\right) \cap \Omega$ for some strongly convex boundary point $p^{\prime} \in \partial \Omega$.

Proof. By Proposition $4, \phi_{j}$ 's converge locally uniformly to a non-constant holomorphic mapping $\phi: \Omega \longrightarrow \Delta_{q}^{\partial \Omega}$. By Theorem 2, one can find a point $z$ close enough to some strongly pseudoconvex boundary point $p^{\prime}$ such that $\phi(z)=q^{\prime}$ for some $q^{\prime} \in \Delta_{q}^{\partial \Omega}$. We have $\phi_{j}(z) \rightarrow q^{\prime}$ non-tangentially as $j \rightarrow \infty$. Therefore by Lemma 4 , one can find $r>0$ and $\left\{p_{j}\right\} \subset \beta_{K}^{\Omega}(z, r)$ such that $\phi_{j}\left(p_{j}\right) \rightarrow q^{\prime}$ normally as $j \rightarrow \infty$. As shown in the proof of Lemma 4, $r$ depends on $\alpha, r=\alpha^{2}$, to be precise. Since we assume $\alpha>0$ is fixed, by Lemma 5 one can choose $z$ close enough to $p^{\prime}$ such that $\beta_{K}^{\Omega}(z, r) \subset B\left(p^{\prime}, \epsilon\right)$.

\section{Boundary accumulation points.}

Proposition 5. Let $\Omega \subset \subset \mathbb{C}^{n}$ be a smoothly bounded convex domain. Suppose $\Delta_{q}^{\partial \Omega}$ is not trivial for some $q \in \partial \Omega$. If there exists $\left\{\phi_{j}\right\} \subset$ Aut $(\Omega)$ such that $\phi_{j}(z) \rightarrow$ $\Delta_{q}^{\partial \Omega}$ nontangentially for all $z \in \Omega$, then $\Delta_{q}^{\partial \Omega}$ is biholomorphic to a complex $m$-ball, where $m$ is the complex dimension of $\Delta_{q}^{\partial \Omega}$ (i.e., real $2 m$ dimensional ball).

Proof. Let $p \in \Omega$ be arbitrarily close to a strongly pseudoconvex boundary point and let $\phi(p)=q \in \Delta_{q}^{\partial \Omega}$. Also denote $p_{j}=\phi_{j}(p)$ and $V=\Delta_{q}^{\partial \Omega}$.

Let $\xi_{1}, \ldots, \xi_{m}$ be $m$ linearly independent complex tangent vectors to $V$ and use the intrinsic measure defined with respect to the complex unit $m$-ball, i.e., $U$ is the complex unit $m$-ball in Definition 2. We may assume $V$ lies in the $z_{2} \ldots z_{m+1}$ plane, where $\operatorname{Re} z_{1}$ is the outward normal direction. Let $\pi$ be the projection mapping of $\mathbb{C}^{n}$ onto the $z_{1} \ldots z_{m+1}$ plane and $\tilde{p}_{j}=\pi\left(p_{j}\right)$. For $j$ large enough, one can find $V^{\prime}$ such that $q \in V^{\prime} \subset \subset V$ and that one can move $V^{\prime}$ into $\Omega$ using the translation mapping that maps $q$ to $\tilde{p}_{j}$. Let $V_{j}^{\prime}$ be the image of such translation mapping of $V^{\prime}$.

We may assume $q=0$. Suppose $p_{j}=\left(a_{1}, \ldots, a_{n}\right), \tilde{p}_{j}=\left(a_{1}, \ldots, a_{m+1}, 0, \ldots, 0\right)$. Consider the holomorphic mapping $f_{j}: \mathbb{C}^{n} \longrightarrow \mathbb{C}^{n}$ defined as $f_{j}(z)=\left(h_{1}(z), \ldots, h_{n}(z)\right)$, where

$$
h_{k}= \begin{cases}z_{k}, & k=1, \ldots, m+1 \\ \frac{a_{k} \cdot \overline{a_{1}}}{\left|a_{1}\right|^{2}} z_{1}, & k=m+2, \ldots, n\end{cases}
$$


Then $f_{j}(0)=0$ and $f_{j}\left(\tilde{p}_{j}\right)=p_{j}$.

We have

$$
\begin{aligned}
\frac{C_{U}^{\Omega}\left(p ;\left(\phi_{j}^{-1} \circ f_{j}\right)_{*}\left(\tilde{p}_{j}\right) \xi_{l}\right)}{K_{U}^{\Omega}\left(p ;\left(\phi^{-1}\right)_{*}(q) \xi_{l}\right)} & \leq \frac{C_{U}^{f_{j}\left(V_{j}^{\prime}\right)}\left(p_{j} ;\left(f_{j}\right)_{*}\left(\tilde{p}_{j}\right) \xi_{l}\right)}{K_{U}^{\Omega}\left(p ;\left(\phi^{-1}\right)_{*}(q) \xi_{l}\right)} \\
& \leq \frac{C_{U}^{V_{j}^{\prime}}\left(\tilde{p}_{j} ; \xi_{l}\right)}{K_{U}^{\Omega}\left(p ;\left(\phi^{-1}\right)_{*}(q) \xi_{l}\right)} \leq \frac{C_{U}^{V^{\prime}}\left(q ; \xi_{l}\right)}{K_{U}^{V}\left(q ; \xi_{l}\right)}
\end{aligned}
$$

where $\xi_{l}$ stands for the set of $m$-vectors, $\xi_{1}, \ldots, \xi_{m}$. Note that $\left(\phi^{-1}\right)_{*} \xi_{j}$ should be interpreted as the pre image vector of $\xi_{j}$, which is well-defined since the rank of $\phi$ is $m$ along $\Delta_{q}^{\partial \Omega}$.

As $j \rightarrow \infty$, one can let $V^{\prime} \rightarrow V$. Then the left hand side approaches 1 , whereas the right hand side is always less than or equal to 1 .

Therefore we have

$$
\frac{C_{U}^{V}\left(q ; \xi_{l}\right)}{K_{U}^{V}\left(q ; \xi_{l}\right)}=1
$$

and hence $V$ is biholomorphic to a complex $m$-dimensional ball.

In the following Theorem, we assume that there exists $\alpha<\infty$ such that (9) holds for all $z$ and in Theorem 4, we will give a proof without the assumption on $\alpha$. The proof of Theorem 3 has its own merit, since is uses the invariant measures to compare the domain $\Omega$ near a strongly convex boundary point and a flat boundary point.

Theorem 3. Let $\Omega \subset \subset \mathbb{C}^{n}$ be a smoothly bounded convex domain. Suppose there exists $\left\{\phi_{j}\right\} \subset$ Aut $(\Omega)$ such that $\phi_{j}(z)$ converges nontangentially to some boundary point for all $z \in \Omega$. We also assume there exists $\alpha<\infty$ such that (9) holds for all $z \in \Omega$. If $q \in \partial \Omega$ is an orbit accumulation point, then $\Delta_{q}^{\partial \Omega}$ is trivial and hence there does not exist a complex variety on $\partial \Omega$ passing through $q$.

Proof. Suppose $\Delta_{q}^{\partial \Omega}$ is not trivial and let $V=\Delta_{q}^{\partial \Omega}$. Let $m$ be the complex dimension of $V$. Since $V$ is convex by Theorem 1, we may assume $V$ lies on a complex $m$-dimensional plane.

We may assume $\nu=(1,0, \ldots, 0)$ is the constant outward unit normal vector along $V$ and $V$ lies in $z_{2} z_{3} \cdots z_{m+1}$ plane after a linear change of coordinates. Let $\pi: \Omega \rightarrow\left\{z_{m+2}=z_{m+3}=\cdots=z_{n}=0\right\}$ be the projection mapping.

By Lemma 6 , one can find a strongly convex boundary point $p^{\prime} \in \partial \Omega$ such that for any $\epsilon>0$, there exists $\left\{p_{j}\right\} \subset B\left(p^{\prime}, \epsilon\right) \cap \Omega$ such that $\phi_{j}\left(p_{j}\right)=q_{j} \rightarrow q^{\prime} \in V$ normally for some $q^{\prime} \in \Delta_{q}^{\partial \Omega}$. Choose $\Omega_{j}$ 's, as a relatively compact exhaustion of $\Omega$, such that $\Omega_{j} \nearrow \Omega$ and that $p_{j} \in \Omega_{j}$ for all $j$. Let $U=\Delta \times \mathbb{B}_{m}$ and choose $m$ linearly independent vectors $\xi_{1}, \ldots, \xi_{m} \in T_{q^{\prime}}^{\mathbb{C}} V$. Since $\partial \Omega$ is geometrically flat along $V$, we have $\xi_{j} \in T_{q^{\prime}-\nu \epsilon}^{\mathbb{C}}(V-\nu \epsilon)$. Hence for $j$ large enough $\xi_{j} \in T_{q_{j}}^{\mathbb{C}}\left(V-\nu\left|q_{j}-q^{\prime}\right|\right)$. Let $\xi_{j}^{\prime}=\left(\phi_{j}^{-1}\right)_{*}\left(q_{j}\right) \xi_{j}$ and $\nu^{\prime}=\left(\phi_{j}^{-1}\right)_{*}\left(q_{j}\right) \nu$

We let $\Gamma_{\epsilon}=\left\{z \in \mathbb{C}: \frac{\pi}{2}+\epsilon<\arg z<\frac{3 \pi}{2}-\epsilon\right\}$ and $H=\{z \in \mathbb{C}: \operatorname{Re} z<0\}$. Then $\Gamma_{\epsilon} \rightarrow H$ as $\epsilon \rightarrow 0$. Let $V_{\epsilon}$ be a subset of $\partial \Omega$ such that $V_{\epsilon} \searrow V$ as $\epsilon \rightarrow 0$. Then we 
have

$$
\begin{aligned}
\frac{C_{U}^{\Omega_{j}}\left(p_{j} ; \nu^{\prime}, \xi_{1}^{\prime}, \ldots, \xi_{m}^{\prime}\right)}{K_{U}^{\Omega}\left(p_{j} ; \nu^{\prime}, \xi_{1}^{\prime}, \ldots, \xi_{m}^{\prime}\right)} & \geq \frac{C_{U}^{\phi_{j}\left(\Omega_{j}\right)}\left(q_{j} ; \nu, \xi_{1}, \ldots, \xi_{m}\right)}{K_{U}^{\Omega}\left(q_{j} ; \nu, \xi_{1}, \ldots, \xi_{m}\right)} \\
& \geq \frac{C_{U}^{\pi\left(\phi_{j}\left(\Omega_{j}\right)\right)}\left(q_{j} ; \nu, \xi_{1}, \ldots, \xi_{m}\right)}{K_{U}^{\Omega}\left(q_{j} ; \nu, \xi_{1}, \ldots, \xi_{m}\right)} \\
& \geq \frac{C_{U}^{\left(H \times V_{\epsilon}\right) \cap W^{\prime}}\left(q_{j} ; \nu, \xi_{1}, \ldots, \xi_{m}\right)}{K_{U}^{\left(\Gamma_{\epsilon} \times V\right) \cap W^{\prime}}\left(q_{j} ; \nu, \xi_{1}, \ldots, \xi_{m}\right)}
\end{aligned}
$$

where $W^{\prime}=W \cap \Omega, W$ an open neighborhood of $V$. In the last inequality we used the inclusion mapping $i: \pi\left(\phi_{j}\left(\Omega_{j}\right)\right) \longrightarrow\left(H \times V_{\epsilon}\right) \cap W^{\prime}$ for the numerator and another inclusion mapping $\tilde{i}:\left(\Gamma_{\epsilon} \times V_{\epsilon}\right) \cap W^{\prime} \longrightarrow \Omega$ for the denominator. The left hand side of (10) is strictly less than 1 since we may assume $p_{j}$ is arbitrarily close to a strongly convex boundary point and $j$ is large enough, whereas the right hand side of (11) approaches 1 since one can let $\epsilon \rightarrow 0$ as $j \rightarrow \infty$, choose $W$ small enough, and $V$ is biholomorphic to a ball by Proposition 5, which leads to a contradition.

REMARK 3. In the proof of Theorem 3 , one can let $U=\mathbb{B}^{m+1}$ instead of $\Delta \times \mathbb{B}^{m}$. In this case we should consider the ratio $K^{\Omega} / C^{\Omega}$. The left hand side of (10) approaches 1 as $p_{j}$ 's approach a strongly pseudoconvex boundary point, whereas the right hand side of (11) is strictly greater than 1 as $q_{j}$ 's approach a flat boundary point. Hence it gives rise to a contradiction.

Additionally, we prove a lemma that shows that if a point converges nontangentially then all the other points must converge non-tangentially in the normal direction.

Lemma 7. Let $\Omega \subset \subset \mathbb{C}^{n}$ be a smoothly bounded convex domain. Suppose $\Delta_{q}^{\partial \Omega}$ is not trivial for some $q \in \partial \Omega$ and that there exists $p \in \Omega$ and $\left\{\phi_{j}\right\} \subset A u t(\Omega)$ such that $\phi_{j}(p) \rightarrow q \in \Delta_{q}^{\partial \Omega}$ non-tangentially. Then $\phi_{j}(a) \rightarrow b \in \Delta_{q}^{\partial \Omega}$ non-tangentially in the normal direction for all $a \in \Omega$ for some $b \in \Delta_{q}^{\partial \Omega}$.

Proof. Let $a \in \Omega$. Since $\Omega$ is complete hyperbolic, we have $d_{K}^{\Omega}(p, a)=r<\infty$ for some $r>0$.

We may assume $q=0$ and the outward normal vector to $\partial \Omega$ along $\Delta_{q}^{\partial \Omega}$ is in the direction of $\operatorname{Re} z_{n}$-axis. Let $p_{j}=\phi_{j}(p)$ and $a_{j}=\phi_{j}(a)$. By Proposition $4, a_{j}$ 's converge to $b \in \Delta_{q}^{\partial \Omega}$ for some $b \in \Delta_{q}^{\partial \Omega}$. Let $p_{j}^{\prime}$ and $a_{j}^{\prime}$ be the projection of $p_{j}$ and $a_{j}$ onto $z_{n}$-axis. Then $p_{j}^{\prime}=\left(0, \ldots, 0, s_{j}\right)$ and $a_{j}^{\prime}=\left(0, \ldots, 0, t_{j}\right)$ for some $s_{j}, t_{j} \in \mathbb{C}$. Let $t_{j}=A_{j} e^{i \alpha_{j}}$ and $s_{j}=P_{j} e^{i \theta_{j}}$. Since $\Omega$ is convex we have Re $s_{j}$, Re $t_{j}<0$.

Since $p_{j} \rightarrow q$ non-tangentially, $p_{j} \in \Gamma_{\alpha}(q)$ for some $\alpha$ for all $j$ large enough. By Lemma 3 , we have $\pi-\theta_{j}<\arccos (1 / \alpha)$ for all $j$ large enough. Hence

$$
\cos \theta_{j}<-1 / \alpha
$$

We have

$$
\infty>r=d_{K}^{\Omega}(p, a)=d_{K}^{\Omega}\left(p_{j}, a_{j}\right) \geq d_{K}^{\Omega}\left(p_{j}^{\prime}, a_{j}^{\prime}\right) \geq d_{K}^{H}\left(s_{j}, t_{j}\right),
$$

where $H=\{z \in \mathbb{C}: \operatorname{Re} z<0\}$. Using the Poincaré distance between two points $z, w \in$ $\Delta$ given by $\ln \left(\frac{|1-w \bar{z}|+|w-z|}{|1-w \bar{z}|-|w-z|}\right)$ and the biholomorphic mapping $f(z)=(z+$ 
1)/(z-1) that maps $H$ to $\Delta$, we get

$$
d_{K}^{H}\left(s_{j}, t_{j}\right)=\ln \left(\frac{\frac{\left|t_{j}+\bar{s}_{j}\right|}{\left|\bar{s}_{j}-1\right|}+\frac{\left|t_{j}-s_{j}\right|}{\left|s_{j}-1\right|}}{\frac{\left|t_{j}+\bar{s}_{j}\right|}{\left|\bar{s}_{j}-1\right|}-\frac{\left|t_{j}-s_{j}\right|}{\left|s_{j}-1\right|}}\right) .
$$

We may assume $\left|s_{j}\right|,\left|t_{j}\right|<1 / 2$. Then we have

$$
\begin{aligned}
d_{K}^{H}\left(s_{j}, t_{j}\right) & \geq \ln \left(\frac{1}{3} \frac{\left|t_{j}+\bar{s}_{j}\right|+\left|t_{j}-s_{j}\right|}{\left|t_{j}+\bar{s}_{j}\right|-\left|t_{j}-s_{j}\right|}\right) \\
& \geq \ln \frac{1}{3}+\ln \left(\frac{\sqrt{1+\cos \left(\theta_{j}+\alpha_{j}\right)}+\sqrt{1-\cos \left(\theta_{j}-\alpha_{j}\right)}}{\sqrt{1+\cos \left(\theta_{j}+\alpha_{j}\right)}-\sqrt{1-\cos \left(\theta_{j}-\alpha_{j}\right)}}\right) \\
& \rightarrow \infty
\end{aligned}
$$

if $\alpha_{j} \rightarrow \pi / 2$. From (12), (13), and (14), we conclude that $a_{j}^{\prime} \rightarrow b \in \Delta_{q}^{\partial \Omega}$ nontangentially for some $b \in \Delta_{q}^{\partial \Omega}$.

REMARK 4. From Lemma 7, it is not hard to see counting the dimensions involved that if there exists a point $p \in \Omega$ such that $\left\{\phi_{j}(p)\right\}$ converges non-tangentially to a boundary point $q \in \partial \Omega$, then $\operatorname{dim} \Delta_{q}^{\partial \Omega}<n-1$, where $n=\operatorname{dim} \Omega$.

In the following theorem we give another proof of Theorem 3 without using the assumption that there exists $\alpha<\infty$ such that (9) holds for all $z \in \Omega$.

ThEOREM 4. Let $\Omega \subset \subset \mathbb{C}^{n}$ be a smoothly bounded convex domain. Suppose there exists $\left\{\phi_{j}\right\} \subset$ Aut $(\Omega)$ such that $\phi_{j}(z)$ converges nontangentially to some boundary point for all $z \in \Omega$. If $q \in \partial \Omega$ is an orbit accumulation point, then $\Delta_{q}^{\partial \Omega}$ is trivial and hence there does not exist a complex variety on $\partial \Omega$ passing through $q$.

Proof. As in the proof of Theorem 3, one can assume $V=\Delta_{q}^{\partial \Omega}$ lies on a complex $m$-dimensional plane, where $m$ is the complex dimension of $V$.

Let the Re $z_{1}$-direction be the outward normal direction along $V$ and $V$ lies on the complex $z_{2} z_{3} \cdots z_{m+1}$ plane.

Let $\Gamma_{\epsilon, r}$ be a wedge domain with radius less than $r$ in $\mathbb{C}$ defined as $\Gamma_{\epsilon, r}=$ $\left\{z \in \mathbb{C}: \frac{\pi}{2}+\epsilon<\arg z<\frac{3 \pi}{2}-\epsilon,|z|<r\right\}$. Choose $p \in \Omega$ close to a strongly pseudoconvex boundary point. Then $\phi_{j}(p) \rightarrow q \in V$ non-tangentially for some $q$. Let $V^{\prime} \subset \subset V$ and $q \in V^{\prime}$. Consider the product domain $\Gamma_{\epsilon, r} \times V^{\prime} \subset \bar{\Omega}$. Let $A_{\epsilon, r}$ be the interior of $\Gamma_{\epsilon, r} \times V^{\prime}$. Let $q=0, p_{j}=\phi_{j}(p)$ and $\tilde{p}_{j}$ be the projection of $p_{j}$ onto the $z_{1} z_{2} \cdots z_{m+1}$-plane, i.e. if $p_{j}=\left(a_{1}, \ldots, a_{n}\right)$, then $\tilde{p}_{j}=\left(a_{1}, a_{2}, \ldots, a_{m+1}, 0, \cdots, 0\right)$. Then $\tilde{p}_{j} \rightarrow q$ nontangentially.

Consider the holomorphic mapping $f_{j}: \mathbb{C}^{n} \longrightarrow \mathbb{C}^{n}$ defined as $f_{j}(z)=\left(h_{1}(z), \ldots, h_{n}(z)\right)$, where

$$
h_{k}= \begin{cases}z_{k}, & k=1, \ldots, m+1 \\ \frac{a_{k} \cdot \overline{a_{1}}}{\left|a_{1}\right|^{2}} z_{1}, & k=m+2, \ldots, n .\end{cases}
$$

Note that $f_{j}$ is the identity mapping when restricted to $V$ and $f_{j}\left(\tilde{p}_{j}\right)=p_{j}$. Since $p_{j} \rightarrow q$ non-tangentially, one can find $\epsilon, r>0$ such that $f_{j}\left(A_{\epsilon, r}\right) \subset \Omega$ assuming $j$ is large enough. 
Let $U=\Delta \times \mathbb{B}_{m}, \xi_{j}$ be the unit vector in the $z_{j}$-direction and $\Omega_{k}$ be the exhaustion of $\Omega$, i.e, $\Omega_{k} \nearrow \Omega$. Then we have

$$
\frac{C_{U}^{\Omega_{k}}\left(p ;\left(\phi_{j}^{-1}\right)_{*}\left(p_{j}\right) \xi_{l}\right)}{K_{U}^{\Omega}\left(p ;\left(\phi_{j}^{-1} \circ f_{j}\right)_{*}\left(\tilde{p}_{j}\right) \xi_{l}\right)} \geq \frac{C_{U}^{\phi_{j}\left(\Omega_{k}\right)}\left(p_{j} ; \xi_{l}\right)}{K_{U}^{\Omega}\left(p_{j} ;\left(f_{j}\right)_{*}\left(\tilde{p}_{j}\right) \xi_{l}\right)} \geq \frac{C_{U}^{A_{\epsilon, r}}\left(\tilde{p}_{j} ; \xi_{l}\right)}{K_{U}^{A_{\epsilon, r}}\left(\tilde{p}_{j} ; \xi_{l}\right)},
$$

where $\xi_{l}$ stands for the set of $m+1$ vectors $\xi_{2}, \ldots, \xi_{m+1}$. Note that the first $(m+1)$ by $(m+1)$ complex Jacobian of $f_{j}$ is the identity and hence $\left(f_{j}\right)_{*} \xi_{l}$ is well-defined for $l=1, \ldots, m+1$. The second inequality for the Carathéodory measure is derived using the projection mapping of $\mathbb{C}^{n}$ onto the $z_{1} z_{2} \ldots z_{m+1}$ plane. For $j$ and $k$ large enough we may assume the projection of $\phi_{j}\left(\Omega_{k}\right)$ is inside $A_{\epsilon, r}$ for some $\epsilon$ and $r$. Note that the Jacobian matrix of the projection is identity along $z_{1} \ldots z_{m+1}$ direction, hence $\xi_{1}, \ldots, \xi_{m+1}$ remain unchanged.

Since $f_{j}$ is the identity along $z_{1}, \ldots, z_{m+1}$ directions, letting $j, k \rightarrow \infty$, we see that the left side of (15) is strictly less than 1, whereas the right hand side converges to 1 as one can let $\epsilon \rightarrow 0$ and $V^{\prime} \rightarrow V$. Hence a contradiction.

\section{REFERENCES}

[1] G. Aladro, The comparability of the Kobayashi approach region and the admissible approach region, Illinois J. Math., 33:1 (1989), pp. 42-63.

[2] S. Fu AND B. Wong, On boundary accumulation points of a smoothly bounded pseudoconvex domain in $\mathbb{C}^{2}$, Math. Ann., 310 (1998), pp. 183-196.

[3] I. Graham, Boundary behavior of the Carathéodory and Kobayashi metrics on strongly pseudoconvex domains in Cn with smooth boundary, Trans. Amer. Math. Soc., 207 (1975), pp. $219-240$.

[4] A. V. Isaev And S. G. Krantz, Domains with non-compact automorphism group: a survey, Adv. Math., 146:1 (1999), pp. 1-38.

[5] K. T. KIM, Domains in Cn with a piecewise Levi flat boundary which possess a noncompact automorphism group, Math. Ann., 292:4 (1992), pp. 575-586.

[6] S. G. Krantz, Function theory of several complex variables. Second edition, The Wadsworth \& Brooks/Cole Mathematics Series. Wadsworth \& Brooks/Cole Advanced Books \& Software, Pacific Grove, CA, 1992.

[7] H. L. Royden, Remarks on the Kobayashi metric, Several complex variables, II (Proc. Internat. Conf., Univ. Maryland, College Park, Md., 1970), pp. 125-137. Lecture Notes in Math., Vol. 185, Springer, Berlin, 1971.

[8] B. Thomas, Thesis, University of California Riverside, 2012

[9] B. Wong, Characterizations of the ball in $\mathbb{C}^{n}$ by its automorhpism group, Invent. Math., 41 (1977), pp. 253-257.

[10] B. Wong, Characterization of the bidisc by its automorphism group, Amer. J. of Math., 117 (1995), pp. 279-288

[11] B. Wong, On complex manifolds with noncompact automorphism groups, Explorations in complex and Riemannian geometry, pp. 287-304, Contemp. Math., 332, Amer. Math. Soc., Providence, RI, 2003. 
